\title{
En majeur ou en mineur ? Tonalités et modalités des incipit romanesques stendhaliens
}

Éric Bordas

\section{Q OpenEdition}

\section{Journals}

Édition électronique

URL : http://journals.openedition.org/recherchestravaux/347

DOI : 10.4000/recherchestravaux.347

ISSN : 1969-6434

Éditeur

UGA Éditions/Université Grenoble Alpes

\section{Édition imprimée}

Date de publication : 15 juillet 2009

Pagination : $37-47$

ISBN : 978-2-84310-146-5

ISSN : 0151-1874

Référence électronique

Éric Bordas, «En majeur ou en mineur? Tonalités et modalités des incipit romanesques stendhaliens », Recherches \& Travaux [En ligne], 74 | 2009, mis en ligne le 28 février 2011, consulté le 08 septembre 2020. URL : http://journals.openedition.org/recherchestravaux/347 ; DOI : https:// doi.org/10.4000/recherchestravaux.347 


\section{En majeur ou en mineur? Tonalités et modalités des incipit romanesques stendhaliens}

«Ne nous embarbouillons pas dans les métaphores», faisait dire Balzac, qui s'y entendait, à un de ses personnages ${ }^{\mathrm{I}}$. Ton et Tonalité sont d'abord et avant tout des mots du vocabulaire acoustique et musical : leur utilisation pour analyser un texte écrit relève donc d'une approche métaphorique transsémiotique des supports d'expression ${ }^{2}$. Ce qui n'est pas un crime, et s'avère peut-être même inévitable voire indispensable pour parler de style, mais ce qui relève tout de même d'une approche analogique et non définitoire des objets33.

$\mathrm{Au}$ sens strict, donc, le «ton» désigne la distance entre deux notes conjointes, plus ou moins variable, ou, pour le dire plus simplement, la hauteur du son définie par rapport à son repère («donner le ton», c'est-à-dire la note repère sur laquelle les musiciens s'accordent). Et il faut savoir qu'on désigne un ton par le nom de la première note de la gamme dont il est formé (un ton d'ut, de ré) - dans ce cas, on parle aussi, et plus souvent, de «tonique». Par métonymie, la «tonalité» recouvre l'ensemble des caractères liés au choix d'une tonique déterminée; on complète l'indication de la tonalité ainsi définie, par celle du «mode», majeur ou mineur, cette disposition particulière des intervalles de la gamme diatonique. C'est ainsi que telle page peut être «en mi mineur», par exemple, soit «dans la tonalité de mi et dans le mode mineur».

I. Illusions perdues, dans Balzac, La Comédie humaine, t. V, Gallimard, «Bibliothèque de la Pléiade», I 976-I 98 I, p. 434.

2. Pour une théorisation de ce geste intellectuel, voir B. Vouilloux, Langages de l'art et relations transesthétiques, Éditions de l'Éclat, I 997.

3. Voir, ici même, la mise au point théorique générale de Ph. Jousset. 
Cette leçon d'harmonie est-elle d'une quelconque utilité pour l'analyse stylistique d'un texte? Il faut le croire, tant la métaphore du ton et du mode est un lieu commun de la critique littéraire - à moins de penser, et l'on y reviendra en conclusion, que la mention musicale ait pour fonction d'épuiser l'analyse et de fermer la discussion au lieu de l'ouvrir.

Le fait est que les analogies transsémiotiques sont troublantes dans leur cohérence : les premières notes jouées donnent le ton de la page à venir, en étant soumises à une qualité globale de production du son qui modalise, en effet, la production et donc l'audition. Tout se joue dans ces premières notes, qui, immédiatement, font entendre une forme qui peut suggérer un contenu affectif (une signification?) - c'est particulièrement évident dans le chant : les premières notes, instrumentales, posent un cadre, un climat, que la voix va développer ${ }^{4}$; la tonalité exprime et le chant dit.

On peut dire exactement la même chose d'un texte écrit : le ou les premiers mots déterminent une réalisation stylistique qui oriente toute la production à venir. Lieu textuel de mise en place des stratégies de séduction poétique, l'incipit est en lui-même l'actant décisif dans la réalisation du style d'une œuvres : il fait entendre, dans son surgissement, dans sa nouveauté, cette présence individuelle qui est style et qui fait sens. Précisément, quand cette présence stylistique est fortement individualisée, voire personnalisée, on dit souvent, par une confusion qui confond métonymie et métaphore, qu'il y a «un ton», immédiatement identifiable, prétendument audible. Or en analyse de l'oral, le ton n'est rien d'autre qu'une variable prosodique que nous ajoutons pour exemplifier la signification de l'énonciation, parfois en dépit du sens de l'énoncé : je peux prononcer telle phrase avec un ton ironique, un ton menaçant, un ton suppliant, qui dira la même chose (redoublement redondant) ou le contraire (antiphrase) que ce que disent les mots. L'incipit donnerait donc la tonalité stylistique de l'œuvre, par l'identification d'une tonique langagière (le choix des mots proposés), tonalité de mode majeur ou mineur selon que le style s'impose immédiatement avec force ou discrétion.

La métaphore musicale a, en effet, le mérite de la clarté : elle remplit parfaitement sa fonction d'intellection et de suggestion d'une réalité indicible transitivement. Elle permet, dans ce cas, de valoriser le phénomène d'apparition

4. Voir les si belles analyses de Barthes du «si naturel» initial du lied Mondnacht de Schumann : «sa simplicité est une insistance : pour bien des morceaux schumanniens, l'étalement tonal a la valeur d'un seul son qui vibre infiniment jusqu'à nous affoler; la tonique n'est pas douée, ici, d'un "évasement cosmique" (comme celui du premier mi bémol de L'Or du Rhin), mais plutôt d'une masse qui pèse, insiste, impose sa solitude jusqu'à l'obsession». — «Aimer Schumann» [1979], dans R. Barthes, Euvres complètes, t. III, Seuil, I 993-I 995, p. Io50.

5. Sur la poétique de l'incipit romanesque, l'étude de référence est celle d'A. Del Lungo, L'Incipit romanesque, Seuil, 2003. 
et de réalisation, la performance, du style comme révélation d'une sensibilité, d'un être-au-monde. Quant à savoir si elle est indispensable... On dirait plutôt «inévitable».

Il n'est pas de «grand écrivain» sans style original. Or l'originalité du style doit se découvrir dès l'épreuve de l'incipit. Donc l'incipit, réalisation majeure d'une poétique des textes, ne saurait être neutre stylistiquement. Un examen de dix incipit romanesques stendhaliens, choisis parmi les textes tout simplement les plus célèbres ${ }^{6}$, montre que, en effet, la grande originalité de l'auteur, comparé à ses références contemporaines', est le soin d'une tonalité, le plus souvent ironique, badine, légère, qui oriente l'énonciation du côté de la narration d'un conte oralisé, contre la lourdeur (balzacienne) du roman. La métaphore s'impose d'elle-même, tant, incontestablement, Stendhal cherche à faire entendre, sinon une voix, ce qui est absolument impossible, du moins un phrasé, un rythme énonciatif personnel dans la diction du récit, un ton donc, qui individualise très fortement le discours produit. Il s'agit là d'un trait de style authentiques.

Les deux exemples les plus nets de ce choix esthétique sont sans doute $L e$ Rouge et le Noir [5] et Lucien Leuwen [6], et leur originalité est d'obtenir cette réalisation tonale par des moyens exclusivement langagiers, et même grammaticaux, très précisément circonscrits. Et dans les deux cas, il se trouve que, comme par hasard, c'est le phénomène appelé en linguistique «modalisation d'auxiliaire verbal» qui concrétise la modalité stylistique du discours, en un mode mineur plein d'ironie : croire ce qui va être raconté, mais ne pas trop prendre les choses au sérieux, tout de même, marquer une distance, de bonne compagnie et de juste hauteur. Le ton juste est aussi et surtout un bon goût, qui fait reconnaître certains préjugés de classe sous la plume du libéral Beyle?.

L'incipit du Ronge [5] est remarquable par la remise en question de la modalité aléthique introduite par le semi-auxiliaire : «La petite ville de Verrières peutpasser pour l'une des plus jolies.» Le ton est immédiat, ironique, soupçonneux, voire narquois : la modalisation réfute l'affirmation, par une antiphrase implicite; si la ville "peut passer pour jolie», c'est, clairement, laisser entendre qu'elle n'est pas jolie aux yeux du narrateur, qui non seulement ne prend pas le lieu

6. Voir le corpus, reproduit en annexe de l'étude : les textes sont présentés dans l'ordre chronologique de rédaction.

7. Voir également en annexe quelques autres incipit d'époque.

8. Voir É. Bordas et Ph. Berthier éd., Stendhal et le style, Presses Sorbonne Nouvelle, 2005 ; F. Spandri, Stendhal. Stile e dialogismo, Macerata, Quodlibet, 2007.

9. Voir "Goût», dans Y. Ansel et al. éd., Dictionnaire de Stendhal, Champion, 2003, p. 304305 . 
commun à son compte, mais laisse entendre que le jugement est fragile. On est déjà dans la polyphonie (discours de l'un versus discours des autres) qui va structurer ce roman des ragots et des vanités ${ }^{10}$, mais selon un angle ambigu, qui suggère sans affirmer, et qui peut échapper à qui lit trop vite - ou à qui n'écoute pas bien : et c'est, du reste, absolument la même chose, ici.

Dans Lucien Leumen [G] ${ }^{11}$, le ton est encore plus immédiatement remarquable, audible, en ce qu'il se réalise par le choix d'une tournure verbale passablement désuète au XIX ${ }^{\mathrm{e}}$ siècle, qui suggère immédiatement un paramètre de réalisation parodique dans le patron stylistique convoqué : «Lucien avait été chassé $[. .$.$] pour s'être allé promener.» L'antéposition du personnel réfléchi de$ l'infinitif de progrédience par rapport au verbe recteur transitif (s'être allé promener versus être allése promener), sur le modèle de la langue classique, qui peut insérer des unités entre le personnel et son verbe («se mal conduire», etc.), réalise un écart stylistique évident - que l'on compare avec tous les autres exemples, [r] à [7’]. On a presque l'impression d'entendre le début d'une fable de La Fontaine, avec hétérométrie construite et rime en [e] : «Lucien Leuwen avait été chassé [décasyllabe]/de l'École polytechnique, [octosyllabe]/pour s'être allé promener [heptasyllabe]", ou d'un conte de Voltaire ${ }^{12}$. La narration est d'emblée posée dans le mode mineur d'un ironique détachement de bon aloi : de toute évidence, tout cela n'est pas bien grave, ou ne mérite pas que l'on fasse semblant d'y attacher une grande importance. La correction trop soutenue de l'énonciation est suspecte : la parodie colore la représentation d'une couche de vernis critique ${ }^{13}$.

L'ironie stylistique de la tournure verbale est ensuite exemplifiée par le complément de temps : «une des célèbres journées de juin, avril, février I 832 ou [18]34». L'ironie n'est pas du tout dans l'antiphrase - l'énoncé est littéral - mais dans l'antithèse entre le sémantisme de l'adjectif «célèbres» et l'hésitation posée par les juxtapositions et la coordination alternative résomptive en «ou» : les journées sont célèbres, mais on ne sait plus trop quand elles ont eu lieu, selon une hésitation de quelques mois et de deux années. Tout cela sent bon l'approximation détachée d'un conteur qui n'aura pas la

ıo. Sur la polyphonie stendhalienne, voir M. Parmentier, Stendhal stratège. Pour une poétique de la lecture, Droz, 2007.

I I. L'histoire du texte de ce roman vient de connaitre une étape décisive, avec l'édition par X. Bourdenet du manuscrit autographe, qui révèle un quasi nouvel incipit, du point de vue rythmique - voir infra, n. 22.

I 2. Sur le goût de Stendhal pour La Fontaine et Voltaire, voir Y. Ansel et al. éd., op. cit., p. 380, 742-745; sur l'auteur des Fables, plus particulièrement, voir J.-P. Collinet, «Stendhal et La Fontaine», Recherches \& Travaux, n 21, Grenoble, i98 I, p. 83-104.

I3. Sur la poétique de la parodie, en particulier dans les récits du XIX ${ }^{\mathrm{e}}$ siècle, voir D. Sangsue, La Relation parodique, José Corti, 2007. 
cuistrerie de vouloir faire concurrence à l'état civil, mais qui bavardera aimablement pour nous divertir.

Curieusement, le ton bavard de Stendhal dans ces deux incipit peut aussi passer par des ajouts, et non par des suppressions pressées. C'est le cas de la détermination référentielle du nom propre : «l'une des plus jolies de la Franche-Comté» [5]. Autre écart stylistique : le français standard, même en I 830, privilégie la construction avec déterminant ø pour les noms géographiques (l'une des plus jolies de Franche-Comte); comme dans le cas du personnel antéposé de [6], la valeur de la construction avec article, d'effet archaïsant, est quasi parodique.

Toutefois, il faut bien dire que les incipit à modalité détachée ironisante ne sont en rien des raretés à cette époque; mais le régime stylistique dominant ne procède pas par semblable individualisation de l'énonciation. On peut, par exemple, comparer ces effets d'ironie stendhaliens à l'ironie assez lourde de Hugo au début de Notre-Dame de Paris [4'] : toute la parodie, chez Hugo, est construite et obtenue par des effets d'accumulation, des précisions chiffrées, et par un rythme ternaire de caricature ${ }^{\mathrm{I}}$. Balzac, dans La Peau de chagrin [3'], ironise, non sur la narration elle-même, comme le fait Stendhal, donc, mais sur les références parisiennes, et son discours critique, modalisant, passe par l'usage d'adverbes d'incidence externe de second degré, qui viennent doubler, en un discours 2, le discours I - quand, chez Stendhal, il y a concomitance absolue des deux discours, et impossibilité de distinguer l'un de l'autre : «conformément à la loi» prépare l'oxymore quasi burlesque de «passion essentiellement imposable».

Une autre façon de faire ainsi entendre un ton à repères prosodiques individuels et oralisant est la mobilisation directe des actants communicationnels de base, locuteur et allocutaire. C’est par un «nous» qui n'est pas une énallage, mais un vrai «nous» de pluriel que s'ouvrent deux des derniers récits de Stendhal : [9] et [1०]. En [9], la relation locuteur-allocutaire est une vraie relation de lecture, avec inférences à des références partagées; en [ Io], l'effet de réel énonciatif est encore plus net, puisque l'inscription du «nous» se fait par rapport à un «je» initial et recteur, véritable axe, en amont, et à un déictique temporel démonstratif «ce soir», en aval. L'énonciation narrative se rapproche ainsi des grands corpus romanesques du XVIII ${ }^{\mathrm{e}}$ siècle, presque toujours à la première personne ${ }^{15}$,

I4. P. Laforgue me répond qu'il ne voit aucune trace d'ironie poétique dans cet incipit, énoncé qu'il veut lire comme une réécriture romantique de l'épique quasi merveilleux des contes et légendes des temps très anciens, à symbole ésotérique chiffré. On peut quand même se demander si le choix d'un hommage à une poétique de l'archaïsme et une herméneutique sacrée n'est pas aussi ironisation du matérialisme contemporain.

I 5. Voir R. Démoris, Le Roman à la première personne, Armand Colin, I 975. 
qui pouvaient encore servir de repères esthétiques à certains contemporains que Stendhal n'aimait guère, ou prétendait ne pas aimer, comme madame de $\operatorname{Duras}^{16}-\operatorname{voir}\left[\mathrm{I}^{\prime}\right]$.

Mais à la différence de ces récits à la première personne, qui introduisent in medias res une action factuelle, les deux seuls incipit à la première personne de Stendhal sont, très manifestement, des ouvertures, là encore, modalisatrices. Il ne s'agit pas de commencer par narrer, d'informer sur ceci ou cela, mais de donner son humeur, d'inscrire une sensibilité dans la prose, de - on y revient toujours - faire entendre un ton, badin, distancié, qui pourra passer pour un style, dans l'absence même d'un style de référence rhétorique (grand style, style oratoire ou autre). Du reste, quand le récit proprement dit, factuel, aura commencé, ces axes énonciatifs disparaitront et l'explicitation individuelle du style par un ton de causerie à la veillée sera refusée - voir la clausule de l'introduction de Lamiel, et sa réponse en écho à cette déclaration initiale qui prend ses aises : «Ainsi, ô lecteur bénévole, adieu; vous n’entendrez plus

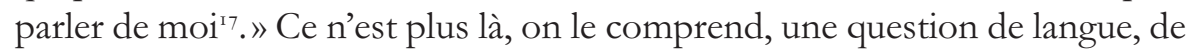
grammaire, et le travail d'analyse ne passe plus par des remarques de forme, de matériau, mais c'est une question de contenu, de substance. Le style, en [9] et en [ $\mathrm{r} \circ]$, n'est plus tant dans la langue que dans le discours.

C'est pourquoi, répétons-le, Stendhal a peu pratiqué l'incipit in medias res au sens strict, contrairement à ses illustres contemporains - voir [2'], [3'], [5'], [6']. Raconter lui plait moins que bavarder, donner son avis. Mais, alors que chez Balzac le bavardage entraîne la digression, et ce parfois dès l'incipit ${ }^{18}$, chez Stendhal le bavardage se traduit par un resserrement concis de la modalisation individualisante, avec le goût brillant de la pointe. Dans notre corpus, on ne voit guère que l'exemple [7], avec ses précisions expansées, son passé simple et son arrière-plan coordonné, qui soit un véritable incipit narratif d'action. Les autres cas sont plus contestables : on peut annexer à cette catégorie le [2], surtout du fait de la dramatisation posée par l'adverbe d'urgence «À peine», mais l'imparfait fait immédiatement comprendre que l'énonciation va aller du côté du rappel analeptique avant de conduire le lecteur à l'événementiel contemporain qui motive la narration. Le [3] est un autre bon exemple d'incipit in medias res, le plus balzacien du corpus, avec son déictique démonstratif, mais, là encore, l'imparfait laisse supposer plus un développement sur

I6. Voir É. Bordas, "“Censurer le style d'une duchesse”. Style et idéologie», dans Ph. Berthier et P.-L. Rey éd., Stendhal journaliste anglais, Presses Sorbonne Nouvelle, 200I, p. I 89 -2I 2.

17. Stendhal, Lamiel, Gallimard, «Folio», I983, p. 53.

I 8. Voir A. Déruelle, Balzac et la digression : une nouvelle prose romanesque, Saint-Cyr-sur-Loire, Christian Pirot, 2004. 
le cadre que sur l'action elle-même, à venir. Quant à l'exemple [4], véritable incipit narratif et romanesque avec le verbe de l'inchoatif par exemple qu'est le verbe perfectif naître, au passé simple, comme il se doit, son efficacité narrative est un peu contrariée par l'analyse d'ethnographie ironique que pose le zeugme "pays de la philosophie et de l'imagination» pour caractériser l'Allemagne. Par ce jugement implicite, il est clair que le narrateur s'efface derrière l'analyste, le moraliste, qui fait, une fois de plus, entendre le son de sa voix.

C'est cette même tendance, cette même esthétique du glissement, du narratif vers l'analytique, et du silence vers le bavardage, qui caractérise l'incipit de $L a$ Chartreuse de Parme [8]. Mais avec une tout autre efficacité. La séquence est en deux phrases, en fait - ou en deux temps, et, pour filer la métaphore musicale, on a envie de dire «en deux mesures». I) «Le is mai i 796, le général Bonaparte fit son entrée à Milan» : c'est l'exemple le plus classique, le plus concis, le plus parfait qui soit, de «récit historique» à la Benveniste; passé simple, nom propre, cadrage spatio-temporel, référence extratextuelle par allusion aux événements historiques attestés dans les livres d'histoire, et, surtout, absence totale d'unités axiologiques individualisantes : les faits, rien que les faits, portés par un rythme rapide et informatif. 2) «à la tête de cette jeune armée qui venait de passer le pont de Lodi, et d'apprendre au monde qu'après tant de siècles César et Alexandre avaient un successeur» : l'ajout de circonstants voit l'énonciation se modifier radicalement avec le pivot du déterminant démonstratif «cette jeune armée qui». Démonstratif + relative : on reconnait le schéma de la très dixneuviémiste détermination par exophore mémorielle ${ }^{19}$, mais, ici, la relative est à l'imparfait, et non au présent, ce qui modifie la représentation, sans changer absolument la pragmatique enclenchée par le tour. Car l’imparfait est, en [8], anaphorique, renvoyant à de l'antériorité; donc le démonstratif qui désigne une réalité placée à sa droite (geste cataphorique) ouvre sur une sollicitation qui lui est antérieure, en un geste, donc, contradictoire (aval spatial, puis amont temporel). Précisément, la contradiction est résolue à condition de comprendre que le geste n'est pas endophorique, mais exophorique et qu'il désigne directement une zone de représentation imaginaire - quoique historique - modalisée par un imparfait d'atténuation modale. C'est d'autant plus net que l'imparfait recteur (le premier des deux) est lui-même en structure de périphrase aspectuelle de proximité conclusive «qui venait de». Le démonstratif attrape le lecteur par la main et l'intègre, non dans la représentation, mais dans la narration de cette représentation. De sorte que, là aussi, se réalise une individualisation du

I9. Voir É. Bordas, «Un stylème dix-neuviémiste? Le déterminant discontinu un de ces... qui...", L'Information grammaticale, $200 \mathrm{I}, \mathrm{n}^{\circ} 90, \mathrm{p} .32-43$. 
discours recteur, qui tire l'énonciation du côté du particulier ${ }^{20}$. Beaucoup plus discrètement qu'en [5] et [6], qui jouaient des repères externes de place de mots ou d'auxiliaires, l'énonciation stendhalienne, en [8], joue d'une désignation directe qui est celle-là même d'une deixis implicite, entièrement intériorisée, proche d'un geste oralisant, mais répondant à une logique de représentation qui n'est ni de l'oral, ni de l'écrit, et n'appartient qu'à la vérité arbitraire d'un récit.

C'est dire que Stendhal invente ses règles et prend le risque, parfois, de certaines confusions, de dérapages, de glissements. Le ton auquel sera ensuite soumis le reste du livre portera les traces de cette poétique de l'instabilité. Et il est très intéressant de constater que ce qui serait le premier incipit romanesque stendhalien développé, soit l'exemple [I], n'est rien d'autre qu'une authentique anacoluthe : quel est, en effet, le statut du second groupe nominal? «une jeune fille habitant un château isolé» ne peut pas être apposé à «Dans une âme parfaitement indifférente» : il n'y a ni coréférence stricte des thèmes («âme, jeune fille»), ni prédication seconde. Bien sûr, la lecture glisse sur la cohérence syntaxique, en rétablissant implicitement un pronom apposé dont le second groupe nominal n'est plus qu'un complément essentiel : «*Dans une âme parfaitement indifférente, [celle d']une jeune fille habitant»; mais c'est là une opération complémentaire, qui contredit, du reste, l'approximation référentielle et caractérisante voulue par une énonciation aussi rapide. Majeur par l'originalité de l'écart, mineur par la poétique de l'implicite, par la vitesse des enchaînements aussi, le mode stendhalien ne ressemble, quoi qu'il en soit, à aucun autre : sa cursivité nerveuse, ses prises de risques, ne pouvaient qu'éloigner à tout jamais son auteur des lenteurs étalées et de la linéarité tranquille de George Sand, par exemple - voir [5'], [7’] -, référence d'une stylistique linguistique des années I 83 I-I 839 .

Cette très rapide lecture de quelques incipit romanesques stendhaliens à la lumière de la métaphore musicale du ton et du mode prouve certainement la cohérence de cette image cognitive, à valeur explicative, pour décrire le rendu stylistique d'une énonciation originale. Comme nous ne savons ni ce qu'est le style, ni dire la musique, nous pouvons toujours parler de musique pour dire l'effet stylistique d'une phrase. Et le référent extralangagier est alors plus une référence d'autorité qu'une vérité quelconque. La métaphore musicale est l'un de ces aveux d'échecs conceptuels que l'analyste tente de retourner

20. C'est une variation originale sur le modèle balzacien qui est le repère de cette poétique du récit, surtout en incipit; voir, par exemple, Sarrasine : «J'étais plongé dans une de ces rêveries profondes qui saisissent tout le monde, même un homme frivole, au sein des fêtes les plus tumultueuses. Minuit venait de sonner à l'horloge de l'Élysée-Bourbon.» 
en victoire intellectuelle, via la caution d'une vague poésie désignative : il n'y a, du reste, ni à la diaboliser, ni à l'exacerber car elle fait partie de ces «métaphores de la vie quotidienne» que les cognitivistes américains distinguent des tropes ornementaux pour en faire des quasi-catachrèses obligées. On ne peut ni parler ni penser sans métaphores ${ }^{21}$. Elle nous aide, incontestablement, à décrire cette originalité d'individuation qui caractérise l'énonciation stendhalienne, laquelle vient nous rappeler, une fois de plus, combien sont poreuses les frontières génériques (romans, récits, autobiographie, etc.) quand un style est, à ce point, dominant de et dans la langue d'un auteur.

\section{Annexe : corpus de l'étude}

[I] Dans une âme parfaitement indifférente, une jeune fille habitant un château isolé, au fond d'une campagne, le plus petit étonnement excite profondément l'agitation. - Ernestine ou la Naissance de l'amour (posthume; rédaction : I 825 ?)

[2] À peine âgé de vingt ans, Octave venait de sortir de l'École polytechnique. - Armance (1827)

[3] C'était un soir du printemps de $182^{*}$. - Vanina Vanini (1 829)

[4] Mina de Vanghel naquit dans le pays de la philosophie et de l'imagination, à Kœnigsberg. - Mina de Vanghel (posthume; rédaction : I 829-1 830)

[5] La petite ville de Verrières peut passer pour l'une des plus jolies de la FrancheComté. - Le Rouge et le noir (1830)

[6] Lucien Leuwen avait été chassé de l'École polytechnique, pour s'être allé promener mal à propos, un jour qu'il était consigné à l'École ainsi que ses camarades, c'était à l'époque d'une des célèbres journées de juin, avril, février I 832 ou [I 8] 34 . - Lucien Leunven (posthume; rédaction : I 834) 22

2I. Voir É. Bordas, Les Chemins de la métaphore, PUF, 2003.

22. Le texte ici cité et commenté est celui publié par X. Bourdenet dans la toute dernière édition du roman (dans Stendhal, Euvres romanesques complètes, t. II, Gallimard, «Bibliothèque de la Pléiade», 2007), qui reproduit fidèlement, pour la première fois, le manuscrit autographe. Rappelons que jusqu'alors Lucien Leuwen était connu dans des éditions philologiquement plus ou moins fiables. Les lecteurs du $\mathrm{xx}^{\mathrm{e}}$ siècle lisaient, en fait, ce roman, presque toujours dans les éditions de H. Debraye ou de H. Martineau. Or la comparaison entre l'incipit de leurs éditions et l'incipit du manuscrit, révélé par X. Bourdenet, découvre des différences profondes. Qu'on en juge. Voici la version Martineau : «Lucien Leuwen avait été chassé de l'École polytechnique pour s'être allé promener mal à propos, un jour qu'il était consigné, ainsi que tous ses camarades : c'était à l'époque d'une des célèbres journées de juin, avril ou février I 832 ou 34.» Martineau supprime la virgule entre "polytechnique» et «pour», qui marque pourtant nettement, dans un geste explicatif, le détachement rythmique du fait et de la cause; il coupe 
[7] Ce fut vers la fin de $183^{*}$ que le général major comte von Landek revint à Kœnigsberg sa patrie; depuis bien des années il était employé dans la diplomatie prussienne. - Le Rose et le vert (I 837)

[8] Le I 5 mai 1796, le général Bonaparte fit son entrée dans Milan à la tête de cette jeune armée qui venait de passer le pont de Lodi, et d'apprendre au monde qu'après tant de siècles César et Alexandre avaient un successeur. - La Chartreuse de Parme (I 839)

[9] Le mélodrame nous a montré si souvent les brigands italiens du XvI e siècle, et tant de gens en ont parlé sans les connaitre, que nous en avons maintenant les idées les plus fausses. - L'Abbesse de Castro (1 839)

[ro] Je trouve que nous sommes injustes envers les paysages de cette belle Normandie où chacun de nous peut aller coucher ce soir. - Lamiel (posthume; rédaction : 1 839)

[I'] J'allais rejoindre à Baltimore mon régiment, qui faisait partie des troupes françaises employées dans la guerre d'Amérique; et, pour éviter les lenteurs d'un convoi, je m'étais embarqué à Lorient sur un bâtiment marchand armé en guerre. - Mme de Duras, Édouard (1 825 )

[2'] La nuit du 24 janvier 1799 était, à Naples, si brillante d'étoiles et si pure, qu'on pouvait découvrir d'une des hauteurs qui dominent la mer tout ce rivage courbé en deux arcs qui s'étend de Pouzzoles à Sorrente. - Latouche, Fragoletta (I 829)

[3'] Vers la fin du mois d'octobre dernier, un jeune homme entra dans le PalaisRoyal au moment où les maisons de jeu s'ouvraient, conformément à la loi qui protège une passion essentiellement imposable. - Balzac, La Peau de chagrin (I 83 I)

[4'] Il y a aujourd'hui trois cent quarante-huit ans six mois et dix-neuf jours, que les Parisiens s'éveillent au bruit de toutes les cloches sonnant à grande volée dans la triple enceinte de la Cité, de l'Université et de la Ville. - Hugo, Notre-Dame de Paris (1831)

[5'] Par une soirée d'automne pluvieuse et fraîche, trois personnes rêveuses étaient gravement occupées, au fond d'un petit castel de la Brie, à regarder brûler les tisons du foyer et cheminer lentement l'aiguille de la pendule. - Sand, Indiana (1 832 )

[6'] Mme Vauquer, née de Conflans, est une vieille femme qui, depuis quarante ans, tient à Paris une pension bourgeoise établie rue Neuve-Sainte-Geneviève, entre le quartier latin et le faubourg Saint-Marceau. - Balzac, Le Père Goriot (I 834-I 835)

le complément de lieu «à l'École» après «était consigné», qui introduit une répétition («École polytechnique, École») intéressante par sa binarité même et par sa discursivité d'anaphore; il ajoute un déterminant totalisant avant «ses camarades», qui introduit un contre-accent inutilement dramatique; il transforme la juxtaposition de la virgule entre la première séquence et la seconde par un double point argumentatif logique. Cela fait beaucoup. Le texte du manuscrit original est infiniment plus rythmé, selon une diction expressive, en effet toute stendhalienne, dans le sens, déjà, de la litote et du sous-entendu. 
[7’] Sur les confins de la Marche et du Berry, dans le pays qu'on appelle la Varenne, et qui n'est qu'une vaste lande coupée de bois de chênes et de châtaigniers, on trouve, au plus fourré et au plus désert de la contrée, un petit château en ruine, tapi dans un ravin, et dont on ne découvre les tourelles ébréchées qu'à environ cent pas de la herse principale. - Sand, Mauprat (1837) 